\title{
Control scheme based on capacitor voltage second-order complex-vector feedforward for LCL-filtered inverter
}

\author{
Linhang Yang ${ }^{1, *}$, Daliang Yang ${ }^{1}$, and Jiahao Chen ${ }^{2}$ \\ ${ }^{1}$ School of Electrical Engineering, Guangxi University, Nanning, China \\ ${ }^{2}$ Liuzhou Power Supply Bureau of Guangxi Power Grid Co., Ltd., Liuzhou, China
}

\begin{abstract}
For an inverter with LCL filter, voltage at the point of common coupling (PCC) is equivalent to the filter capacitor voltage in practical applications. However, the PCC voltage background harmonics will cause the distortion of grid current. Some problems exist in the traditional methods for harmonics suppression, such as insufficient phase margin, increasing design complexity and computation burden. Thus, a scheme of second-order complex-vector feedforward (SOCVF) of capacitor voltage is proposed in this article. First, based on the vectorial principle, the active unit vectors of capacitor voltage were calculated. Then, its fundamental component was obtained by the SOCVF-function. Furthermore, the references of the grid current were obtained with the instantaneous power theory. Hence, the proposed scheme helps effectively dampen the LCL resonance to make the system stable, and works well at suppressing the injected grid current distortion simultaneously. Simulation and experimental results validated the effectiveness of the proposed scheme.
\end{abstract}

\section{Introduction}

The high penetration of distributed power generation systems (DPGS) work well in alleviating the problems of environmental pollution and energy shortage. More and more grid-connected inverters are installed as the interface between the DPGS and the grid, and greatly improve the quality of injected grid current [1-2]. In the applications, the LCL filter is generally adopted due to its better ability in harmonics attenuation and smaller size compared with the L filter [3]. However, a resonance peak exists in the LCL filter, which makes the system unstable [4]. Thus, the method called filter capacitor current proportional feedback (FCCPF) has been used widely due to its relatively simple structure [5]. Nevertheless, the additional sensors for sensing the capacitor current are needed, which increases the cost of the system.

As the grid impedance exists in practical applications, what's more, the inverter is usually connected to the PCC by a step-up transformer. So that the grid voltage is difficult to directly measure, and the PCC voltage is often used instead [6-7]. Meanwhile, since the voltage of LCL-grid-side inductor is much smaller than the PCC voltage, it can be

\footnotetext{
* Corresponding author: yanglinhang@st.gxu.edu.cn
} 
considered that the PCC voltage is equivalent to the voltage of filter capacitor [8]. However, with more and more non-linear devices connect to the PCC, the injected grid current distorts because of the increasing of the background harmonics [9]. To suppress the serious distortion of the grid voltage, the total harmonic distortion (THD) of injected grid current must not exceed 5\% according to the regulation IEC 61000-3-4. Thus, the harmonics attenuation ability should be considered sufficiently of the controller design.

For suppressing the current distortion, extensive studies have been conducted. In [10], based on the traditional proportional-resonant (PR) control, a structure of multiple paralleled resonators is proposed to suppress the multiple harmonics of the injected grid current. However, it increases the computation burden and design complexity of the control system. In contrast, a structure based on the combination of proportional integral (PI) controller and the repetitive controller (RC) is proposed in [11], which has smaller computation burden and a simpler structure. The two methods above are both to boost the loop gain of current of the fundamental and background harmonic frequencies. However, it might make system instable due to the insufficiency of phase margin [12]. To address this issue, the scheme called grid-voltage feedforward (GVF) is adopted [13], which can suppress the multiple current harmonics simultaneously and do not effects the loop gain of current. However, for an LCL-filtered inverter, the GVF actually realizes by the capacitor voltage of LCL according to previous analysis, which still affects the stability of the system.

This article proposes a scheme of second-order complex-vector feedforward (SOCVF) of capacitor voltage. It can not only suppress the distortion of the injected grid current, but also dampen the LCL resonance. As a result, the system becomes stable, and has superior harmonics attenuation ability. Finally, the performance of the proposed scheme is verified by the results of digital simulation and experiment.

\section{Conventional active damping scheme of LCL-filtered inverter}

The three-phase four-wire LCL-filtered grid-connected inverter topology is shown in Fig. 1. The structure of the converter bridge is neutral point clamped (NPC). The filter is LCL-type, which consists of the NPC-side inductor $L_{1}$, the filter capacitor $C_{\mathrm{f}}$ and the grid-side inductor $L_{2} . C_{\mathrm{dc} 1}, C_{\mathrm{dc} 2}$ are split DC capacitors, and point-N is linked to the neutral-point of the clamping diode $D_{1}, D_{2}$ [14]. $u_{\mathrm{g}}$ is the grid voltage, and $L_{\mathrm{g}}$ is the grid impedance. $u_{x}(x=\mathrm{a}, \mathrm{b}, \mathrm{c})$ is the output voltage of the converter bridge. $u_{\mathrm{cf}}$ is the voltage of $C_{\mathrm{f}}$, and $i_{\mathrm{cf}}$ is the current flowing through $C_{\mathrm{f}} . i_{1}$ is the current flowing through $L_{1}$, and $i_{2}$ is the current injecting to the grid.

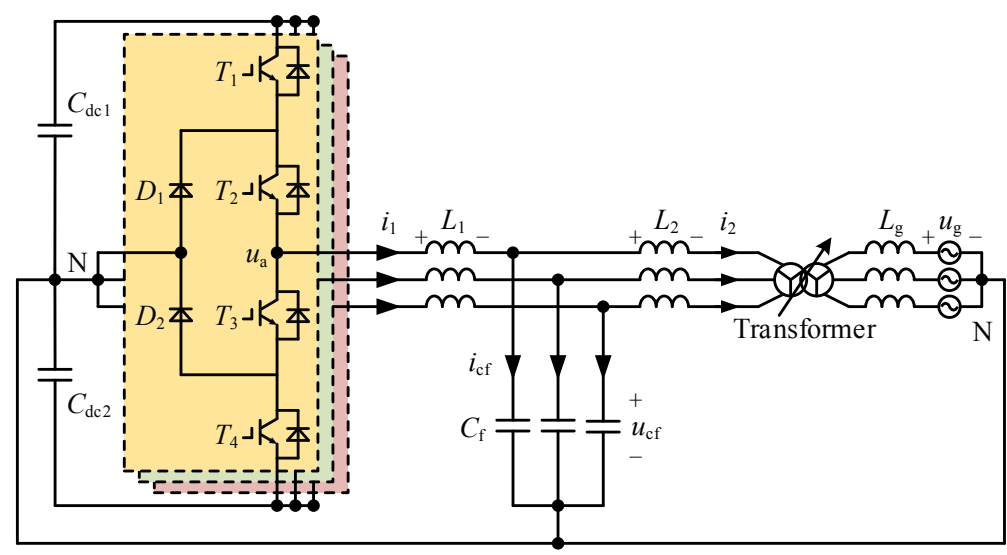

Fig. 1. Main Circuit of three-phase four-wire LCL-filtered grid-connected inverter. 
Assuming that all components are ideal, and the $L_{\mathrm{g}}$ is neglected. According to Fig. 1 , the $s$-domain state equation of the LCL-filtered grid-connected inverter can be obtained as:

$$
\left[\begin{array}{c}
u_{x}(s) \\
u_{\mathrm{cf}}(s) \\
i_{1}(s)
\end{array}\right]=\left[\begin{array}{c}
u_{\mathrm{cf}}(s) \\
u_{\mathrm{g}}(s) \\
i_{2}(s)
\end{array}\right]+s\left[\begin{array}{ccc}
L_{1} & 0 & 0 \\
0 & L_{2} & 0 \\
0 & 0 & C_{\mathrm{f}}
\end{array}\right]\left[\begin{array}{c}
i_{1}(s) \\
i_{2}(s) \\
u_{\mathrm{cf}}(s)
\end{array}\right]
$$

As seen, it is a 3rd-order system, and exists LCL resonance peak that may lead to the oscillation of both $i_{1}$ and $i_{2}$. Conventionally, the active damping method of FCCPF is used to dampen the resonance peak.

The control block diagram of FCCPF is given in Fig. 2. Where, $i_{x}^{*}(x=\alpha, \beta)$ is the reference of grid current $i_{2 x}, H_{\mathrm{i} 2}$ is the sensor gain of $i_{2 x}$, and $H_{\mathrm{ic}}$ is the coefficient of FCCPF. $G_{\mathrm{i}}(s)$ is the current regulator with quasi-proportional-resonant (qPR) structure. $Z_{\mathrm{L} 1}=s L_{1}$, $Z_{\mathrm{L} 2}=s L_{2}, Z_{\mathrm{C}}=1 / s C_{\mathrm{f}}$ are the impedance of $L_{1}, L_{2}$, and $C_{\mathrm{f}}$ respectively. $K_{\mathrm{PWM}}=U_{\mathrm{dc}} / U_{\text {tri }}$ is the transfer function of NPC converter bridge, where $U_{\mathrm{dc}}$ is the DC voltage, and $U_{\text {tri }}$ is the amplitude of the triangular carrier. $\mathrm{e}^{-1.5 s T_{s}}$ is the 1.5-sampling-period delay that consists of calculation and PWM delay [15].

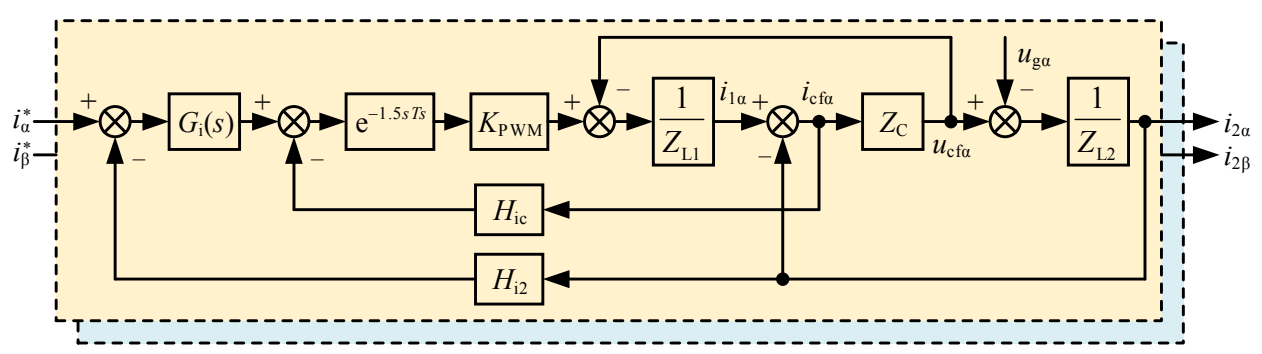

Fig. 2. Control block diagram of FCCPF active damping.

By applying the superposition theorem, the expression of $i_{2 x}(s)$ can be obtained from the structure relationship in Fig. 2 as:

$$
i_{2 x}(s)=\frac{G_{\mathrm{o}}(s)}{H_{\mathrm{i} 2}+H_{\mathrm{i} 2} G_{\mathrm{o}}(s)} i_{x}^{*}(s)-\frac{G(s)}{1+G_{\mathrm{o}}(s)} u_{\mathrm{g} x}(s)=i_{2 \times 1}(s)+i_{2 \times 2}(s)
$$

where $G_{\mathrm{o}}(s)$ is the current loop gain, $G_{\mathrm{o}}(s)$ and $G(s)$ are expressed as:

$$
\begin{aligned}
& G_{\mathrm{o}}(s)=\frac{H_{\mathrm{i} 2} K_{\mathrm{PWM}} \mathrm{e}^{-1.5 s T_{s}} G_{\mathrm{i}}(s)}{s^{3} L_{1} L_{2} C_{\mathrm{f}}+s^{2} L_{2} C_{\mathrm{f}} H_{\mathrm{ic}} K_{\mathrm{PWM}} \mathrm{e}^{-1.5 s T_{s}}+s\left(L_{1}+L_{2}\right)} \\
& G(s)=\frac{s^{2} L_{1} C_{\mathrm{f}}+s C_{\mathrm{f}} H_{\mathrm{ic}} K_{\mathrm{PWM}} \mathrm{e}^{-1.5 s T_{s}}+1}{s^{3} L_{1} L_{2} C_{\mathrm{f}}+s^{2} L_{2} C_{\mathrm{f}} H_{\mathrm{ic}} K_{\mathrm{PWM}} \mathrm{e}^{-1.5 s T_{s}}+s\left(L_{1}+L_{2}\right)}
\end{aligned}
$$

Although combining the qPR regulator with FCCPF can effectively dampen the LCL resonance, as seen in (2) that $i_{2 x}(s)$ is composed of the static tracking component $i_{2 \times 1}(s)$ and the disturbance component $i_{2 \times 2}(s)$. In the ideal grid, it may only exist the grid current steady-state errors. When the grid operates in non-ideal state, the current reference $i_{x}^{*}$ may distort, and further leads to the severe distortion of the injected grid current.

\section{Capacitor voltage second-order complex-vector feedforward}

To address the drawbacks of the conventional methods, this article proposes a scheme of second-order complex-vector feedforward (SOCVF) of capacitor voltage. First, the active unit vectors of capacitor voltage are calculated according to the vectorial principle. Then, its fundamental component is obtained by the SOCVF-function. Finally, the grid current 
commands are obtained according to the instantaneous power theory. The detailed principles of the scheme will be described in the following sections.

\subsection{Calculation of capacitor voltage unit composite vector}

The instantaneous power is constant in a three-phase AC system, and the distribution of the active and reactive components indicates the actual operation state of a system. Thus, if the instantaneous voltage is transformed into active and reactive components, the active and reactive components of the grid current can be independently controlled in $d q$ frame [16].

According to the analysis in Section 1, the PCC voltage $u_{\mathrm{pcc}}$ is equal to the filter capacitor voltage $u_{\mathrm{cf}}$, thus the phase information of $u_{\mathrm{cf}}$ can be used as reference phase of $i_{x}^{*}$. Define the direction of the active voltage composite vector $\boldsymbol{u}_{\mathrm{cfs}}$ of $u_{\mathrm{cfx}}(x=\mathrm{a}, \mathrm{b}, \mathrm{c})$ to be consistent with the $d$-axis, lagging of $90^{\circ}$ with respect to the reactive voltage composite vector $\boldsymbol{u}_{\mathrm{cfs}}^{\prime}$. The vector relationship is shown in Fig. 3.

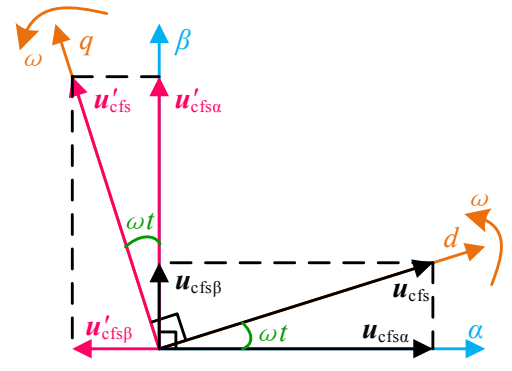

Fig. 3. Active and reactive voltage vector based on $\alpha \beta$ reference frame.

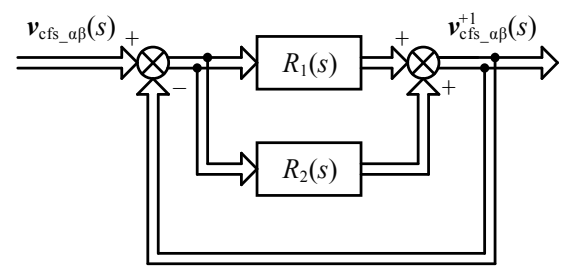

Fig. 4. Realization of SOCVF-function based on unit negative feedback.

Where $\omega t$ is the phase information of $u_{\mathrm{cf}}$, and $\boldsymbol{u}_{\mathrm{cfs} \alpha}$ and $\boldsymbol{u}_{\mathrm{cfs} \beta}$ are the components of $\boldsymbol{u}_{\mathrm{cfs}}$ in each axis of $\alpha \beta$ frame. Thus, according to Fig. 3, the unit vectors $\boldsymbol{v}_{\mathrm{cfs} \alpha}, \boldsymbol{v}_{\mathrm{cfs} \beta}$ of $\boldsymbol{u}_{\mathrm{cfs}}$, and the unit vectors $\boldsymbol{v}_{\mathrm{cfs} \alpha}^{\prime}, \boldsymbol{v}_{\mathrm{cfs} \beta}^{\prime}$ of $\boldsymbol{u}_{\mathrm{cfs}}^{\prime}$ can be derived as follows:

$$
\left\{\begin{array}{l}
\boldsymbol{v}_{\mathrm{cfs} \alpha}=\frac{\boldsymbol{u}_{\mathrm{cfs} \alpha}}{\sqrt{\boldsymbol{u}_{\mathrm{cfs} \alpha}^{2}+\boldsymbol{u}_{\mathrm{cfs} \beta}^{2}}}=\frac{\boldsymbol{u}_{\mathrm{cfs} \alpha}}{\left|\boldsymbol{u}_{\mathrm{cfs}}\right|}=\cos \omega t \\
\boldsymbol{v}_{\mathrm{cfs} \beta}=\frac{\boldsymbol{u}_{\mathrm{cfs} \beta}}{\sqrt{\boldsymbol{u}_{\mathrm{cfs} \alpha}^{2}+\boldsymbol{u}_{\mathrm{cfs} \beta}^{2}}}=\frac{\boldsymbol{u}_{\mathrm{cfs} \beta}}{\left|\boldsymbol{u}_{\mathrm{cfs}}\right|}=\sin \omega t
\end{array},\left\{\begin{array}{l}
\boldsymbol{v}_{\mathrm{cfs} \alpha}^{\prime}=\frac{\boldsymbol{u}_{\mathrm{cfs} \alpha}^{\prime}}{\sqrt{\boldsymbol{u}_{\mathrm{cfs} \alpha}^{\prime 2}+\boldsymbol{u}_{\mathrm{cfs} \beta}^{\prime 2}}}=\frac{\boldsymbol{u}_{\mathrm{cfs} \alpha}^{\prime}}{\left|\boldsymbol{u}_{\mathrm{cfs}}^{\prime}\right|}=\boldsymbol{v}_{\mathrm{cfs} \beta}=\sin \omega t \\
\boldsymbol{v}_{\mathrm{cfs} \beta}^{\prime}=\frac{\boldsymbol{u}_{\mathrm{cfs} \beta}^{\prime}}{\sqrt{\boldsymbol{u}_{\mathrm{cfs} \alpha}^{\prime 2}+\boldsymbol{u}_{\mathrm{cfs} \beta}^{\prime 2}}}=\frac{\boldsymbol{u}_{\mathrm{cfs} \beta}^{\prime}}{\left|\boldsymbol{u}_{\mathrm{cfs}}^{\prime}\right|}=-\boldsymbol{v}_{\mathrm{cfs} \alpha}=-\cos \omega t
\end{array}\right.\right.
$$

\subsection{Principle of second-order complex-vector feedforward function}

As is known, the second-order scalar filter in analog electronic circuits can suppress the high-frequency harmonics, and the typical transfer function is expressed as:

$$
A(s)=\frac{\omega_{\mathrm{k}}}{s^{2}+2 \zeta \omega_{\mathrm{n}} s+\omega_{\mathrm{n}}^{2}}
$$

where $\zeta$ is the damping ratio, which is usually 0.707 in order to optimize the dynamic performance. $\omega_{\mathrm{n}}$ is the natural angular frequency, and $\omega_{\mathrm{k}}$ is the adjustable gain.

However, when the negative sequence component of the input signal is relatively large, the output signal becomes distorted by adopting the typical transfer function of equation (6). Thus, for achieving the suppression of the high-frequency harmonics and extracting the fundamental component of the input signal simultaneously, a zero point of $s_{\mathrm{z}}=-j \omega_{\mathrm{o}}$ is 
substituted into the numerator of (6). Let $\omega_{\mathrm{n}}=\omega_{0}, \omega_{\mathrm{k}}=\zeta \omega_{\mathrm{o}}$, where $\omega_{\mathrm{o}}$ is the power angular frequency. Then the proposed SOCVF-function can be expressed as:

$$
A(s)=\frac{\zeta \omega_{\mathrm{o}}\left(s+j \omega_{\mathrm{o}}\right)}{s^{2}+2 \zeta \omega_{\mathrm{o}} s+\omega_{\mathrm{o}}^{2}}=\frac{R_{1}(s)}{1+R_{1}(s)+R_{2}(s)}
$$

where $R_{1}(s)$ and $R_{2}(s)$ are expressed as:

$$
R_{1}(s)=\frac{\zeta \omega_{\mathrm{o}}}{s-j \omega_{\mathrm{o}}}, R_{2}(s)=\frac{\zeta \omega_{\mathrm{o}}}{s+j \omega_{\mathrm{o}}}
$$

Fig. 4 shows a diagram of the realization of SOCVF-function based on unit negative feedback. Further, as shown in Fig. 5, the dashed part is the realization of SOCVF-function in scalar form, where $K$ is the adjustable gain, and its value is $2 \zeta$.

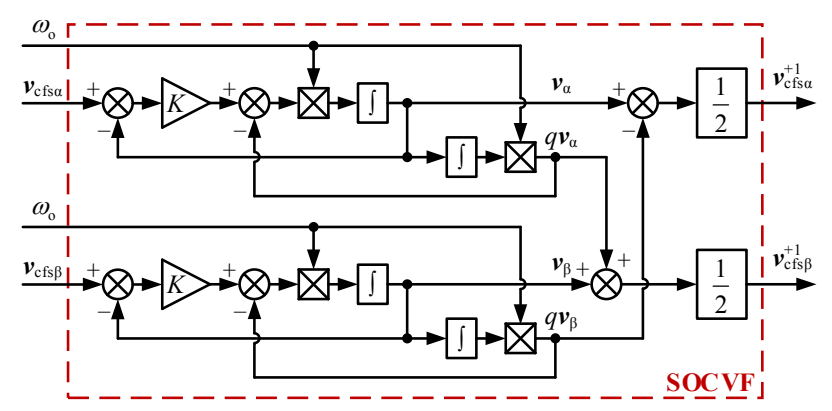

Fig. 5. Realization of SOCVF-function in scalar form.

The input-output relationship of Fig. 5 can be described in (9), further the amplitudefrequency characteristics of $H_{\mathrm{d}}(s)$ and $H_{\mathrm{q}}(s)$ can be derived as shown in (10), where $\omega$ is the input signal angular frequency. As seen in (10), when $\omega$ is taken value as $\omega_{0}$, the value of amplitude gain is 1 , and the phase offset is 0 . Thus the fundamental of input signal can be tracked without static error. Meanwhile, the amplitude gain will be greatly attenuated with the increment of $\omega$, that is, the high-frequency harmonics can be effectively suppressed.

$$
\begin{aligned}
& \left\{\begin{array}{l}
\boldsymbol{v}_{-\alpha \beta}(s)=\frac{2 \zeta \omega_{\mathrm{o}} s}{s^{2}+2 \zeta \omega_{\mathrm{o}} s+\omega_{\mathrm{o}}^{2}} \cdot \boldsymbol{v}_{\mathrm{cfs} \alpha}=H_{\mathrm{d}}(s) \cdot v_{\mathrm{cfs} \alpha} \\
q \boldsymbol{v}_{-\alpha \beta}(s)=\frac{2 \zeta \omega_{\mathrm{o}}^{2}}{s^{2}+2 \zeta \omega_{\mathrm{o}} s+\omega_{\mathrm{o}}^{2}} \cdot \boldsymbol{v}_{\mathrm{cfs} \beta}=H_{\mathrm{q}}(s) \cdot \boldsymbol{v}_{\mathrm{cfs} \beta}
\end{array}\right. \\
& \left\{\begin{array}{l}
\left|H_{\mathrm{d}}(j \omega)\right|=\frac{\omega}{\sqrt{\left(K \omega_{\mathrm{o}} \omega\right)^{2}+\left(\omega_{\mathrm{o}}^{2}-\omega^{2}\right)}}\left|H_{\mathrm{q}}(j \omega)\right| \\
\angle H_{\mathrm{d}}(j \omega)=\arctan \left(\frac{\omega_{\mathrm{o}}^{2}-\omega^{2}}{K \omega_{\mathrm{o}} \omega}\right)=\angle H_{\mathrm{q}}(j \omega)+90^{\circ}
\end{array}\right.
\end{aligned}
$$

Furthermore, combining the principles in Section 2.1 and the instantaneous power theory, the current reference under $\alpha \beta$-frame can be obtained as follow:

$$
\left[\begin{array}{l}
i_{\alpha}^{*} \\
i_{\beta}^{*}
\end{array}\right]=\frac{1}{\left|\boldsymbol{u}_{\mathrm{cfs}}\right|}\left[\begin{array}{cc}
\boldsymbol{v}_{\mathrm{cfs} \alpha}^{+1} & \boldsymbol{v}_{\mathrm{cfs} \alpha}^{+1} \\
\boldsymbol{v}_{\mathrm{css} \beta}^{+1} & \boldsymbol{v}_{\mathrm{cfs} \beta}^{\prime+1}
\end{array}\right]\left[\begin{array}{l}
p \\
q
\end{array}\right]=\left[\begin{array}{cc}
\boldsymbol{v}_{\mathrm{cfs} \alpha}^{+1} & \boldsymbol{v}_{\mathrm{cfs} \alpha}^{+1} \\
\boldsymbol{v}_{\mathrm{cfs} \beta}^{+1} & \boldsymbol{v}_{\mathrm{css} \beta}^{+1}
\end{array}\right]\left[\begin{array}{l}
i_{\mathrm{d}}^{*} \\
i_{\mathrm{q}}^{*}
\end{array}\right]=\left[\begin{array}{cc}
\boldsymbol{v}_{\mathrm{cfs} \alpha}^{+1} & \boldsymbol{v}_{\mathrm{cfs} \beta}^{+1} \\
\boldsymbol{v}_{\mathrm{cfs} \beta}^{+1} & -\boldsymbol{v}_{\mathrm{cfs} \alpha}^{+1}
\end{array}\right]\left[\begin{array}{c}
i_{\mathrm{d}}^{*} \\
i_{\mathrm{q}}^{*}
\end{array}\right]
$$

where $i_{\mathrm{d}}^{*}$ is the active current reference, and $i_{\mathrm{q}}^{*}$ is the reactive current reference. $p$ is the instantaneous active power, and $q$ is the instantaneous reactive power.

Therefore, according to the principles described above, the control block diagram of proposed scheme of SOCVF of capacitor voltage is shown in Fig. 6 . Where $H_{\mathrm{v}}$ is the capacitor voltage sensor gain, and $u_{\mathrm{m} \alpha}, u_{\mathrm{m} \beta}$ are the modulation signals of NPC. 


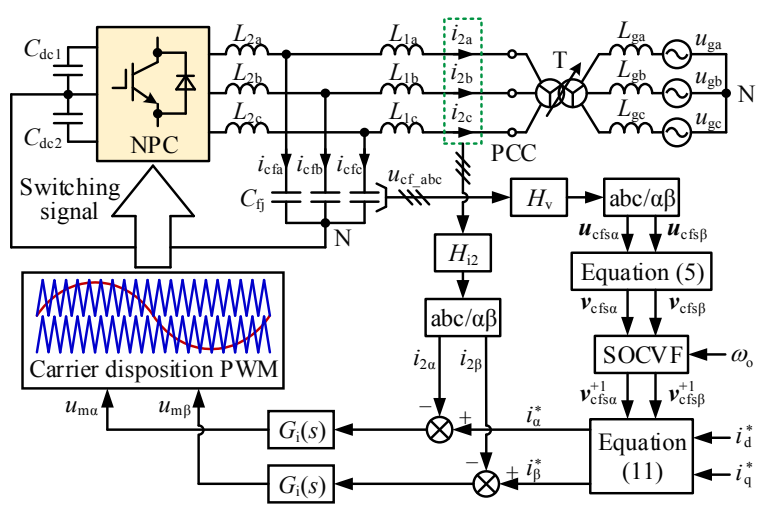

Fig. 6. Capacitor voltage SOCVF control block diagram.

\section{Simulation results}

In order to investigate the feasibility of the proposed scheme, a three-phase four-wire LCL grid-connected inverter system simulation model is carried out in MATLAB/Simulink, and the parameters are shown in Table 1. In addition, for testing the harmonics rejection ability of the proposed scheme, the actual measured PCC voltage data shown in Table 2 are used in simulation. As seen in Table 2, the three-phase PCC voltage in the actual grid is not only unbalanced, but also contains background harmonics. Notably, Table 2 only shows the harmonic order within 15 th due to the measuring limitation of the equipment.

Table 1. Simulation model parameters.

\begin{tabular}{|c|c|c|c|c|c|}
\hline Parameter & Symbol & Value & Parameter & Symbol & Value \\
\hline DC voltage & $U_{\mathrm{dc}}$ & $780 \mathrm{~V}$ & $\begin{array}{l}\text { Grid current } \\
\text { sensor gain }\end{array}$ & $H_{\mathrm{i} 2}$ & 1 \\
\hline DC capacitance & $C_{\mathrm{dc1} 1}, C_{\mathrm{dc} 2}$ & $7800 \mu \mathrm{F}$ & $\begin{array}{l}\text { Capacitor current } \\
\text { feedback coefficient }\end{array}$ & $H_{\text {ic }}$ & 0.5 \\
\hline $\begin{array}{c}\text { PCC voltage (Line-to-line } \\
\text { RMS voltage) }\end{array}$ & $u_{\text {pcc_line }}$ & $380 \mathrm{~V}$ & $\begin{array}{l}\text { Capacitor voltage } \\
\text { sensor gain }\end{array}$ & $H_{\mathrm{v}}$ & 1 \\
\hline Fundamental frequency & $f_{\mathrm{o}}$ & $50 \mathrm{~Hz}$ & Inverter-side inductor & $L_{1}$ & $400 \mu \mathrm{H}$ \\
\hline Sampling frequency & $f_{\mathrm{s}}$ & $15.2 \mathrm{kHz}$ & Filter capacitor & $C_{\mathrm{f}}$ & $20 \mu \mathrm{F}$ \\
\hline Parameters of $G_{\mathrm{i}}(s)$ & $K_{\mathrm{pi}}, K_{\mathrm{ri}}$ & $2.5,500$ & Grid-side inductor & $L_{2}$ & $60 \mu \mathrm{H}$ \\
\hline
\end{tabular}

Table 2. Fundamental and harmonics of voltage at point of common coupling.

\begin{tabular}{|c|c|c|c|c|c|c|c|c|c|}
\hline \multicolumn{2}{|c|}{ Harmonic order } & Fundamental & 3 & 5 & 7 & 9 & 11 & 13 & 15 \\
\hline \multirow{2}{*}{ Phase-A } & RMS value (V) & 233.7 & 5.3 & 5.2 & 2.8 & 2.1 & 1.3 & 1.2 & 0.9 \\
\hline & Phase angle (deg) & 2 & 51 & -146 & -3 & 87 & -94 & -136 & 60 \\
\hline \multirow{2}{*}{ Phase-B } & RMS value (V) & 230.0 & 4.5 & 4.3 & 1.0 & 1.7 & 2.9 & 0.8 & 0.5 \\
\hline & Phase angle (deg) & -123 & 50 & -5 & -97 & -256 & 32 & -188 & -278 \\
\hline \multirow{2}{*}{ Phase-C } & RMS value (V) & 263.8 & 5.9 & 6.0 & 1.2 & 2.8 & 1.2 & 0.4 & 1.2 \\
\hline & Phase angle (deg) & -238 & -312 & -258 & -313 & -263 & -242 & -158 & -316 \\
\hline
\end{tabular}


Setting the grid current reference value of $i_{\mathrm{d}}^{*}=10 \mathrm{~A}, i_{\mathrm{q}}^{*}=0 \mathrm{~A}$, Fig. 7 and Fig. 8 respectively show the simulation waveforms of steady-state grid current with the active damping scheme of FCCPF and the proposed scheme. As seen in Fig. 7, when employing the FCCPF scheme, the grid current is stable though, it is distorted obviously with a THD of $8.64 \%$, which exceeds the industry standard of $5 \%$. When employing the proposed scheme, as seen in Fig. 8, the distortion of $i_{2}$ is almost suppressed with the THD reducing to $2.15 \%$. The simulation results above are consistent with the analysis in Section 3.

Furthermore, Fig. 9 shows the phase-A transient-state simulation waveforms for testing the resonant-damping performance of the proposed method. Obviously, the PCC voltage and grid current of phase-A become stable when adopting the proposed method at the moment of $0.105 \mathrm{~s}$, indicating the effectiveness of LCL resonance damping.

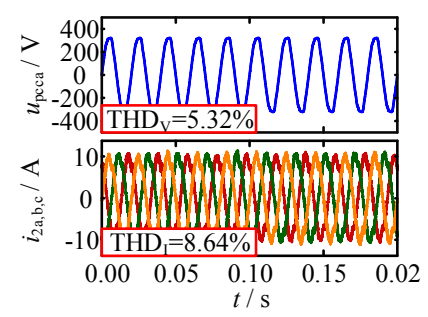

Fig. 7. Simulation results of steady-state grid current by FCCPF scheme.

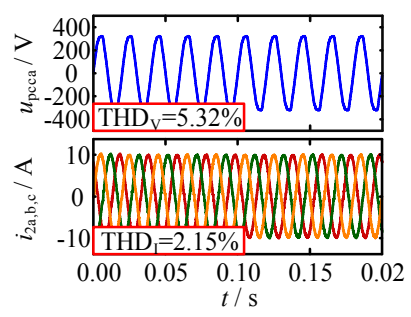

Fig. 8. Simulation results of steady-state grid current by proposed scheme.

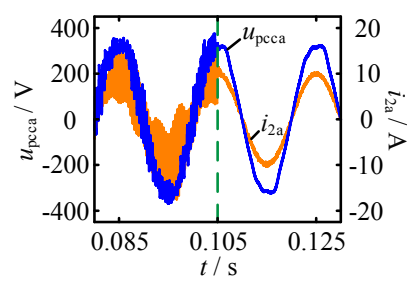

Fig. 9. Simulation results of resonant-damping performance by proposed method.

\section{Experimental results}

For further verifying the proposed scheme, an experimental prototype has been fabricated. The 32-bit floating-point dual-core DSP (TI TMS320F28377D) is used for implementing the control algorithms, and the dead time of the PWM pulse signal is set to $3 \mu \mathrm{s}$. A oscilloscope of TPS-2024 is used to observe the waveforms, and a power quality analyzer of Fluke-435 is used to analysis power quality of $u_{\mathrm{pcc}}$ and $i_{2}$. Notably, severe LCL resonance may occur in experiment, so the following experiments are all conducted at a lower voltage class for safety. Thus, the PCC voltage in Tab. 1 is adjusted to $50 \mathrm{~V}$, the DC voltage is adjusted to $120 \mathrm{~V}$, and the others are the same as simulation. The experimental waveforms are shown in Fig. 10, Fig. 11 and Fig. 12.

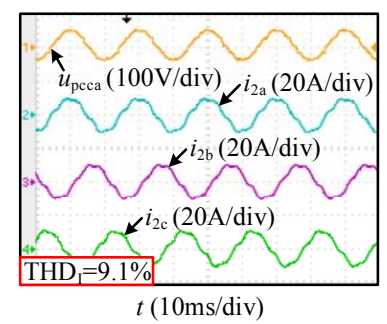

Fig. 10. Experimental results of steady-state grid current by FCCPF scheme.

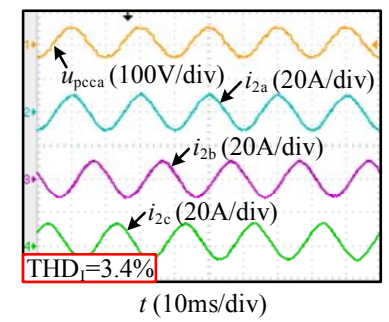

Fig. 11. Experimental results of steady-state grid current by proposed scheme.

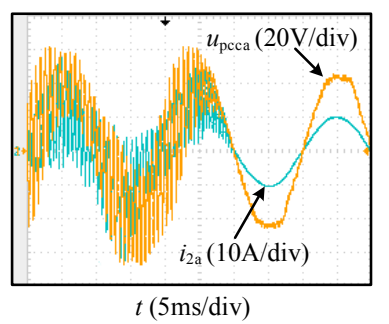

Fig. 12. Experimental results of resonant-damping performance by proposed method.

As seen in Fig. 10, with the active damping scheme of FCCPF, the injected grid current distorts severely with a THD of $9.1 \%$ due to the background harmonics. When employing the proposed scheme, $i_{2}$ becomes highly sinusoidal, as seen in Fig. 11. And the THD drops to $3.4 \%$, indicating the effective harmonics rejection ability of the SOCVF scheme. Then Fig. 12 shows the transient-state experimental waveforms of phase-A for testing the 
resonant-damping performance of SOCVF scheme. It is clear that the resonance of the PCC voltage and grid current are well dampened after a short time, verifying the effectiveness for damping LCL resonance of the proposed scheme. Thus, the results above are consistent with the simulation.

\section{Conclusion}

In this article, a control scheme of capacitor voltage second-order complex-vector feedforward is proposed to suppress the injected grid current distortion of the LCL-type grid-connected inverter caused by the background harmonics. Meanwhile, the LCL resonance can be effectively dampen. In contrast to other methods, it reduces the system complexity and computational burden. In addition, compared with the conventional method of FCCPF, the proposed scheme achieves active damping of the LCL resonance without additional capacitor current sensors, thereby significantly saving the cost in the industrial applications. Although the proposed SOCVF scheme seems more suitable for practice, further research is still needed for its stability analysis and effectiveness under more complex operating conditions.

\section{References}

1. C. Xie, D. Liu, K. Li, et al. Passivity-based design of repetitive controller for LCL-type grid-connected inverters suitable for microgrid applications[J]. IEEE Trans. on Power Electronics, 36, 2420-2431 (2021)

2. X. Wu, Y. Wang, X. Chen, et al. Sequence impedance model and interaction stability research of three-phase grid-connected inverters with considering coupling effects[J]. Proceedings of the CSEE, 40, 1605-1617 (2020)

3. E. Rodriguez-Diaz, F.D. Freijedo, J.M. Guerrero, et al. Input-admittance passivity compliance for grid-connected converters with an LCL filter[J]. IEEE Trans. on Industrial Electronics, 66, 1089-1097 (2019)

4. T. Dragičević, C. Zheng, J. Rodriguez, et al. Robust quasi-predictive control of LCLfiltered grid converters[J]. IEEE Trans. on Power Electronics, 35, 1934-1946 (2020)

5. C. Bao, X. Ruan, X. Wang, et al. Design of grid-connected inverters with LCL filter based on PI regulator and capacitor current feedback active damping[J]. Proceedings of the CSEE, 32, 133-142+19 (2012)

6. Q. Liu, Y. Li, S. Hu, et al. A transformer integrated filtering system for power quality improvement of industrial DC supply system[J]. IEEE Trans. on Industrial Electronics, 67, 3329-3339 (2020)

7. Q. Liu, Y. Li, L. Luo, et al. Power quality management of PV power plant with transformer integrated filtering method[J]. IEEE Trans. on Power Delivery, 34, 941949 (2019)

8. T. Fang, X. Zhang, C. Huang, et al. Control scheme to achieve multiple objectives and superior reliability for input-series-output-parallel LCL-type grid-connected inverter system[J]. IEEE Trans. on Industrial Electronics, 67, 214-224 (2020)

9. D. Pan, X. Ruan, X. Wang, et al. Analysis and design of current control schemes for LCL-type grid-connected inverter based on a general mathematical model[J]. IEEE Trans. on Power Electronics, 32, 4395-4410 (2017) 
10. C. Xie, X. Zhao, K. Li, et al. Multirate resonant controllers for grid-connected inverters with harmonic compensation function[J]. IEEE Trans. on Industrial Electronics, 66, 8981-8991 (2019)

11. M. Lyu, et al. Analysis and design of PI plus repetitive control for grid-side converters of direct-drive wind power systems considering the effect of hardware sampling circuits[J]. IEEE Access, 8, 87947-87959 (2020)

12. J. Xu, S. Bian, Q. Qian, et al. Grid current feedforward based phase-locked loop for sin-gle-phase-inverters in weak grid case[J]. Proceedings of the CSEE, 40, 2647-2657 (2020)

13. W. Li, X. Ruan, D. Pan, et al. Full-feedforward schemes of grid voltages for a threephase LCL-type grid-connected inverter[J]. IEEE Trans. on Industrial Electronics, 60, 2237-2250 (2013)

14. H. Xiao, X. Wang, X. Zhang, et al. State-of-the-art and future trend of transformerless photovoltaic grid-connected inverters $[\mathrm{J}]$. Proceedings of the CSEE, 40, 1038$1054+1397$ (2020)

15. Y. He, X. Wang, X. Ruan, et al. Hybrid active damping combining capacitor current feedback and point of common coupling voltage feedforward for LCL-type gridconnected inverter[J]. IEEE Trans. on Power Electronics, 36, 2373-2383 (2021)

16. D. Yang, N. Wu, L. Yin, et al. Natural frame control of single-phase cascaded Hbridge multilevel converter based on fictive-phases construction[J]. IEEE Trans. on Industrial Electronics, 65, 3848-3857 (2018) 